\title{
Kernos
}

Revue internationale et pluridisciplinaire de religion grecque antique

$10 \mid 1997$

Varia

\section{La Macédoine et les rites d'initiation}

\section{Pierre Brulé}

\section{OpenEdition}

\section{Journals}

Édition électronique

URL : http://journals.openedition.org/kernos/670

DOI : $10.4000 /$ kernos. 670

ISSN : 2034-7871

\section{Éditeur}

Centre international d'étude de la religion grecque antique

\section{Édition imprimée}

Date de publication : 1 janvier 1997

Pagination : 319-330

ISSN : 0776-3824

\section{Référence électronique}

Pierre Brulé, «La Macédoine et les rites d'initiation », Kernos [En ligne], 10 | 1997, mis en ligne le 12 avril 2011, consulté le 24 septembre 2020. URL : http://journals.openedition.org/kernos/670 ; DOI : https:// doi.org/10.4000/kernos. 670 


\title{
Revue des Livres
}

\section{Compte rendu critique}

\section{La Macédoine et les rites d'initiation ${ }^{1}$}

\author{
par Pierre Brulé (Université de Rennes II)
}

S'il est un domaine où la rareté de la documentation spécifique pose d'épineux problèmes aux historiens de la Grèce antique, c'est bien celui des initiations et des rites de passage de la jeunesse. Tout accroissement de la documentation requiert donc un examen attentif, la chose est banale. Ce qui l'est peut-être moins est de prendre suffisamment garde au fait qu'en raison même du caractère quantitativement limité des sources, tout apport significatif risque de modifier passablement l'image qu'on s'est peu à peu forgée de ces institutions. C'est bien à ce double titre que ce nouveau volume des Meletèmata, un volume qui prendra place au rang des plus précieux de cette passionnante série, mérite notre attention. Non que les documents publiés (ou republiés) ici soient très spectaculaires - ils feraient même plutôt dans la modestie -, mais parce que, mis en série avec sagacité par M.B. Hatzopoulos en compagnie d'autres documents déjà connus dont l'A. propose de nouvelles interprétations, ils éclairent d'une lumière entièrement nouvelle les rites de la jeunesse en Macédoine et en Thessalie (remettant ainsi en cause les interprétations anciennes); et l'ensemble permet, par contrecoup, d'approfondir certaines réflexions sur ces mêmes rites dans d'autres régions de Grèce. L'apport n'est d'ailleurs pas seulement d'ordre géographique, mais engage aussi l'histoire : ces traces macédoniennes et thessaliennes impliquent évidemment l'existence d'un passé des institutions de la jeunesse commun à ces régions et au reste de la Grèce.

Le titre du livre risque de tromper un peu; on n'y trouvera pas en effet un traitement en continu du sujet. L'ouvrage ${ }^{2}$ se présente plutôt comme une série de sept

1 A propos de Miltiade B. HATZOPOULos, Cultes et rites de passage en Macédoine, Athènes, Centre de recherches de l'antiquité grecque et romaine. Fondation nationale de la recherche scientifique, 1994. 1 vol. 17,5 × 24,5 cm, 166 p., 36 pl, 2 cartes (Meletèmata, 19). Diffusion De Boccard. ISBN : 960-07094-86-7.

2 Quelques fautes témoignent surtout d'erreurs de saisie. Laissons-les. Je me contenterai de signaler qu'il vaudrait mieux Élis qu'Élide p. 31; que, p. 35, il faut lire: « ne peut pas ne pas évoquer... ». Enfin, entre autres preuves que l'on pourrait trouver hors du contexte de ce livre, le fait que l'on «voue» des garçons et des filles (kouroi et kourai) à une Héra appelée Akrata à Pérachora (p. 35-36) implique que le déterminant joue un rôle cultuel ; il ne s'agit donc pas d'une épithète, mais d'une épiclèse (p. 36) (mème remarque pour Throsia, p. 33). 
dossiers, chacun étant centré généralement sur la publication ou la republication de documents épigraphiques ${ }^{3}$; l'ensemble est suivi d'un appendice qui traite de la localisation du sanctuaire de Déméter et Koré près de Létè ainsi que des fouilles inédites qu'y avait pratiquées N. Kotzias en 1936. Voyons rapidement le contenu de ces dossiers.

1- «De Thessalie en Macédoine » (p. 25-40). M.H. fait retour sur le sens de deux inscriptions conservées aux musées de Volo et de Larissa; la première, publiée par A. Wilhelm en $1880^{4}$ et reprise dans les $I G$ (IX 2, 1123 Kern). M.H. en rapproche d'autres documents publiés en 1984 par A. Tziafalias dans une revue difficilement accessible Thessaliko Hémérélogio (6 [1984], p. 177-208; 7 [1984], p. 193-234). Ces textes, dont les plus anciens remontent à la première partie du $\mathrm{III}^{\mathrm{e}}$ siècle, tous republiés ici par M.H., ont ceci de particulier de comporter une forme du participe $v \varepsilon \beta \varepsilon v ́ \sigma \alpha \sigma \alpha$. Ce rapprochement entre documents thessaliens et macédoniens permet de revenir sur le sens à attribuer à ce verbe et de préciser ainsi le sujet de ces textes.

2 - «Déméter et la Belle Déesse à Létè » (p. 41-54). Nous nous transportons ensuite à Létè en Mygdonie, à proximité de Thessalonique, pour y étudier deux inscriptions inédites du site découvertes par N. Kotzias et copiées par Ch. Edson; elles sont conservées au musée de Thessalonique et proviennent du sanctuaire extra muros de Déméter (v. appendice). Elles contiennent elles aussi ce même participe rare ou une forme dérivée, mais datent selon l'A. du IV ${ }^{\mathrm{e}}$ siècle. Ce dossier constitue donc le prolongement du premier.

3- « Le dromos en Macédoine » (p. 55-62). Cette partie est consacrée à deux autres inscriptions du même lot copiées et estampées par Ch. Edson (mais restées inédites), elles sont rapprochées d'une inscription inédite de Béroia. Elles ont toutes en commun de mentionner un dromos (avec course à pied [pezôn] ou à cheval [bippôn ou bippou]).

4- « Dionysos, Agrios, Érikryptos, Pseudanor» (p. 63-72). Des affranchissements de Béroia, le premier déjà connu (p. 63) et les autres récemment découverts sur une colonne remployée dans l'ancienne « cathédrale » de Béroia, citent des épiclèses intéressantes de Dionysos : le Sauvage, le Faux Homme, le Très Caché.

5- «Imberbe et Travesti » (p. 73-86). Ne comporte pas de document nouveau, mais se présente comme le prolongement interprétatif du dossier précédent.

6- «La guerre et la chasse » (p. 87-112); c'est le dossier qui correspond le mieux au titre de l'ouvrage; il traite des « initiations et rites de passage des jeunes macédoniens », d'abord à travers les sources littéraires. Mais ce qui retiendra le plus l'attention c'est le retour sur la célèbre fresque de la «Tombe de Philippe II » en y pointant le traitement qui s'y trouve fait du costume des personnages : de la nudité au port de la ceinture. L'A. tire aussi parti de deux inscriptions relatives au culte d'Héraklès Kynagidas à Béroia qui nous renseignent sur les kynégoi, pour rétablir la séquence initiatique des jeunes Macédoniens (ou plutôt de l'élite de ceux-ci).

7- «Initiations, consécrations et affranchissements» (p. 113-120). Il s'agit de mettre en exergue cette singularité des sanctuaires extra muros de la Macédoine occi-

3 Un aspect non négligeable de l'intérêt que l'on trouve à cet ouvrage (et plus généralement à cette collection) est de mettre sous nos yeux des documents publiés dans des revues absentes de nos bibliothèques.

4 Je corrige une erreur dans la date p, 25 n. 2. 
dentale, à savoir que les divinités qui y sont vénérées sont des courotrophes et sont destinatrices d'affranchissements par consécration.

On le voit à ce rapide relevé, les documents sont d'une grande diversité, leur provenance aussi, et ce n'est pas sur le terrain de l'épigraphie thessalienne ni surtout macédonienne que je me hasarderai à discuter avec un connaisseur comme M.H.. Mais l'aspect paradoxal de l'affaire c'est que, malgré le caractère fort éclaté de la documentation mise en ouvre, les questions d'histoire religieuse et d'histoire sociale posées par l'ensemble du dossier sont à la fois en petit nombre et fondamentales. Ce sont quelques-unes de ces questions - celles qui concernent les filles, essentiellement - que je me contenterai d'examiner un peu longuement ici, en me plaçant dans une perspective qui permette les comparaisons avec le reste du dossier hellénique des initiations de la jeunesse féminine. Pour des raisons de compétences, je laisserai complètement de côté une partie importante de l'ouvrage : tout ce qui concerne la guerre, la chasse, la fresque de Vergina et les basilikoi kunegoi.

\section{Faire-sa-jeunesse (dossiers 1 et 2 )}

La série de textes utilisés par M.H. provient de Thessalie et de Macédoine (Musée de Volo, de Larissa et de Thessalonique). Ce qui les rassemble, c'est qu'ils contiennent tous une forme quelconque du participe ne(b)eusa( $n$ )sa ou d'un de ses dérivés nouvellement connus par ces textes (epinebeusa(n)sa, tageusa(n)sa, arcbineusa(n)sa). Ce sont pour la plupart des dédicaces dont la structure est banale et remarquablement constante : « $X$ (fille d'Y) nebeusasa dédie l'objet en question (stèle, statue...) à une Artémis » (plusieurs fois Artémis Throsia). La fille en question n'est normalement pas l'auteur réel de la dédicace (c'est le plus souvent une très grande probabilité, c'est parfois certain), c'est un homme qui joue ce rôle. L'ensemble des textes est homogène (on regrette l'absence de certaines indications d'estimation de date) ${ }^{5}$. Toute la question que pose le dossier est de savoir ce qu'avait donc fait la dédicante (ou celle au nom de qui est faite la dédicace) qui ne(b)eusa( $n$ )sa.

C'est un des principaux apports de cet ouvrage que d'établir le sens de ce participe. M.H. propose judicieusement de corriger ce qui, depuis un article de P. Clément en $1934^{6}$, a constitué jusqu'à aujourd'hui sur ce point l'opinion commune. P. Clément y reprenait une suggestion de Hiller von Gaertringen faite à $O$. Kern et que celui-ci a citée dans son édition des $I G$ (texte de Volo, le premier connu, ici, p. 25) sur un texte d'abord publié par A. Wilhelm; cette suggestion proposait de considérer le participe ne(b)eusasa comme dérivant d'un verbe équivalent à celui que les Athéniens utilisaient pour désigner le service des ourses de Brauron (et de Mounichie) : $\alpha \rho \kappa \tau \varepsilon v ́ \sigma \alpha \sigma \alpha$. L'analogie de forme entre arkteuein et ne(b)euein (fallacieuse, on va le voir) invitait donc à voir dans le rite évoqué par l'inscription connue alors un rite zoologique. Dans cette perspective, il ne restait plus qu'à trouver l'animal que faisaient ces filles. Hiller le suggérait fortement en proposant d'écrire neb(r)eusasa : la forme verbale aurait dérivé selon lui du nom du faon : veßpós. Ces filles auraient donc "fait-le-faon ". Telle est l'interprétation développée par P. Clément (et ses suivants...).

5 Ainsi pour la plus importante p. 26, p. 37

6 New Evidence for the Origin of the Ipbigeneia Legend, in $A C, 3$ (1934), p. 393-409.

$7 \operatorname{MDAI}(A), 15(1880)$, p. $303, \mathrm{n}^{\circ} 13$. 
Ce dernier fondait son raisonnement sur la première inscription connue, prove-

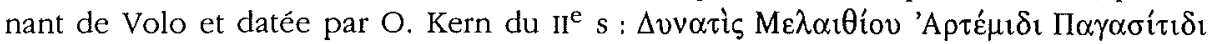
$v_{\varepsilon} \beta \varepsilon v ́ \sigma \alpha[\sigma \alpha$. Mais aussi sur un second texte plus récemment connu et provenant de Larissa, qu'il publiait à cette occasion parallèlement à N.I. Giannopoulos et A.S. Arvanitopoulos. Je dois témoigner du fait que si l'article de Clément fut connu (comme le prouve l'examen des citations dans les études subséquentes), il n'en est pas allé de même pour ceux (antérieurs) des savants grecs ${ }^{8}$. Dans l'ensemble du dossier, c'est cette seconde inscription qui est la plus complète et la plus riche. Donnons-la donc pour aider à restituer le commentaire de l'A.

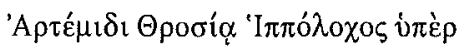

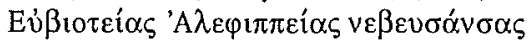 $\lambda u ́ \tau \rho \alpha$}

\section{Le participe}

Ce nouveau texte, produit cinquante ans après celui de $\mathrm{A}$. Wilhelm (puis $I G$ ), avait beau confirmer l'existence d'une forme nebeusa( $n$ )sa sans rbô, P. Clément n'en jugeait pas moins impossible que ce participe dérivât d'autre chose que du substantif nebros ${ }^{9}$ 'faon'. Niant ainsi ce qui semble pourtant l'hypothèse la plus simple et à laquelle il avait d'ailleurs pensé (et qu'il pouvait trouver chez Arvanitopoulos) : à savoir qu'un verbe

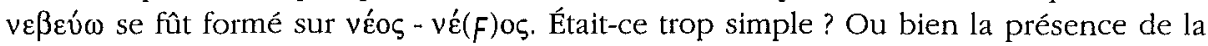
biche dans le mythe d'Iphigénie n'entraînait-elle pas pour lui comme pour ses lecteurs ultérieurs une surdétermination de sens ? Toujours est-il que tous ceux qui ont eu à connaître ensuite de ce dossier ont suivi P. Clément, d'autant plus volontiers que, précisément, son commentaire s'enrichissait à partir de cette lecture erronée d'un parallèle séduisant avec le mythe d'Iphigénie. M.H. a beau jeu de prendre clément et tous ses suivants ${ }^{10}$, dont je fais partie, en flagrant délit d'à peu près. Sur ce premier point en effet le doute n'est pas permis. La forme verbale nebeusa(n)sa ne saurait dériver de nebros 'faon', ni d'une forme *neb-antérieure, mais bien de neos - nea. De là, l'A. induit que celle ${ }^{11}$ qui se trouve ainsi désignée " appartient à une classe d'âge participant à un cycle initiatique. Le participe nebeusa(n)sa désignerait dans cette hypothèse la jeune fille ayant accompli ce cycle, dont la fin était peut-être marquée par un rite de sortie. Cette hypothèse deviendra certitude, ajoute-t-il, si nous sommes en mesure de justifier du point de vue linguistique la forme nebeuô et de fournir des parallèles de son emploi comme terme technique dans un contexte analogue » (p. 32).

Un avis divergent de celui de P. Clément aurait pourtant dû mettre éditeurs et commentateurs en éveil, celui de d'A.S. Arvanitopoulos ${ }^{12}$. Au plan linguistique, il avait

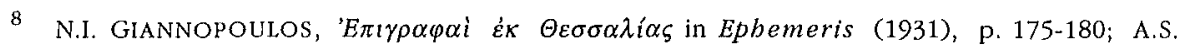

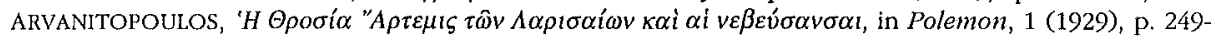
512.

9 Pour expliquer la chute du rbô, il faisait soit appel à un phénomène phonétique, soit en faisant remonter et la forme et le substantif nebros à une racine neb- (p. 403-404).

10 Dont P. Roussel et R. Flacelì̀re, S.G. COLE, K. DOWDEN et moi-même.

11 La forme n'est partout et toujours que féminine.

12 C'est à se demander aujourd'hui qui l'a lu. 
proposé de faire dériver nebeuô de ne(F)os (nous y reviendrons). Cet adjectif prenant, là comme en d'autres circonstances (je fais allusion aux utilisations anthropologiques, sociales, voire politiques de neoi), un sens technique que M.H. compare à ceux d'epbebos ou de partbenos (p. 31), c'est-à-dire un sens précis ${ }^{13}$ dans la nomenclature des classes d'âge. Au plan religieux ou, même, plus généralement, culturel, Arvanitopoulos avait judicieusement exhibé à l'appui de cette interprétation une notice d'Hésychius définissant de la façon suivante des filles qu'on appelait des neai, des

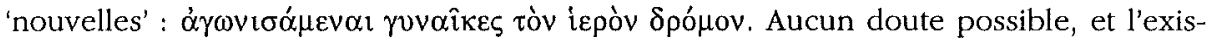
tence d'une entrée vé $\alpha \imath$ dans le glossaire et le contenu même de cette définition doivent faire considérer le terme comme un terme technique. Gunaikes doit être pris ici, je crois, non dans un sens connotant un quelconque âge de la vie (femmes adultes, mariées), mais dans celui de genre féminin. Un usage du mot qui n'est pas particulier à cette définition. Les néai d'Hésychius ce sont, dans le genre féminin, celles qui se trouvent à l'âge où se pratique la « course sacrée ».

Comme l'écrit M.H., « le parallèle lexical le plus courant de nebeuô est offert par son correspondant masculin, pourrait-on dire ${ }^{14}$, le verbe epbebeuô... dérivé du substantif epbebos. Mais, ajoute-t-il, il est aujourd'hui possible de fournir deux exemples du parallèle phonétique exact du verbe nebeuô en macédonien, idiome qui présente depuis les époques les plus reculées des affinités singulières avec la Thessalie $»(\mathrm{p}, 42$ ). M.H. produit alors quelques inscriptions inédites en provenance de la cité de Létè et conservées au Musée de Thessalonique (p. 44). Elles présentent deux intérêts: l'un de vocabulaire et l'autre de date.

Elles conservent une forme composée de ne(F)euô : arcbineusasai, un bapax au sens parallèle à celui d'autres formes dérivées : epineulbleusassa (p. 29) et tageusansa (p. 30) qui, elles, se trouvent dans les dédicaces thessaliennes (toutes deux pour

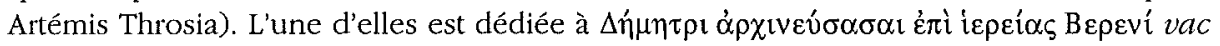

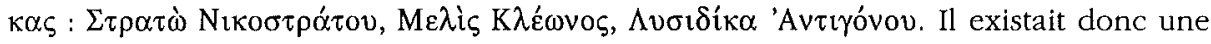
hiérarchie à l'intérieur de ces groupes de filles (aussi bien en Thessalie qu'en Macédoine) et celles qui sont citées là étaient les « cheftaines » de trois de ces groupes. En ce qui concerne ces groupes, M.H. reprend pour Létè une hypothèse que j'avais formulée pour Athènes à propos des ourses et des canéphores, et selon laquelle le recrutement de ces desservantes se faisait dans le cadre des divisions civiques de la cité (comme les éphèbes, par tribu); cette division en trois groupes de filles reproduirait donc celles du corps civique de la cité. ${ }^{15}$

L'autre intérêt de ces nouveaux documents réside dans leur date qui remonte au milieu du $\mathrm{IV}^{\mathrm{e}}$ siècle. Ceci prouve que ne(F)euô a été utilisé en même temps en

13 En cette matière, le fait que le terme revête ici un sens technique précis n'empêche pas de concevoir qu'il prenne ailleurs un autre sens technique précis.

14 Il y aurait à revenir sur la part d'inexactitude qui s'attache à ce rapprochement : être éphèbe c'est être pubère depuis deux ans; pourquoi ces deux ans d'écart entre garçons et filles ?

15 Je suis frappé par le fait que nombre de ces dédicaces sont inscrites sur un petit décor sculpté assez semblable, imitant la forme d'un temple (M.H. parle de naïskos) (2 dédicaces de la p. 30; celle des $I G$ ) (les autres (p. 29) sont des stèles ou des fragments de stèles). Si l'on exclut l'inscription pour Eubiotéta (p. 26), qui appartient à une base de statue et dont le contenu est un peu hors normes, on ne peut compter pour rien cette évocation fréquente d'un décor « religieux » dans le message. Lier l'endroit évoqué - le temple de la divinité protectrice de celles qui nebeusasai - avec le stage plus ou moins long auquel elles ont dû participer ne me semble pas très aventureux. 
Macédoine et en Thessalie. Citons M.H. pour la conséquence historique que l'on peut en tirer : "Cette constatation, ainsi que la diversité des formes, selon les lois de la phonétique des dialectes thessalien et macédonien respectivement... écartent l'éventualité d'un emprunt et indiquent très probablement un héritage commun remontant à la préhistoire des peuples hellénophones ${ }^{16}$.

Revenant à ce fameux participe, on se dit alors que, compte tenu de son caractère temporel, le nebeusa( $n$ )sa des inscriptions pourrait bien désigner un état (biologique / sexuel / social) que l'on pouvait définir clairement, que cet état doive être mis en rapport avec la puberté ou avec le mariage. Son usage au passé dans les dédicaces indiquerait un accomplissement, un accomplissement sanctionné au terme d'un rituel tel que celui que suggère la notice d'Hésychius, ou bien un autre. Publiant plus loin (p. $55 \mathrm{sq}$.) des inscriptions provenant elles aussi du sanctuaire de Létè et mentionnant un dromos, M.H. choisit (sans mentionner la part d'hypothèse de l'enchaînement) la participation des filles à un biéros dromos. Si tel est le cas, une 'course sacrée' pourrait donc solenniser la césure temporelle pour des 'nouvelles' qui nebeusasan. Notons néanmoins qu'en toute rigueur, rien, dans le dossier, n'indique clairement la place précise par un tel rituel dans la vie de cette Eubiotéia ou de cette Dynatis. Avant ou après le mariage?

Quoi qu'il en soit de ce rapprochement (qui n'est qu'un rapprochement, les neai d'Hésychius pouvant évidemment n'avoir aucun rapport précis avec la Thessalie et la Macédoine), la documentation « externe » est bien suffisante pour étayer cette hypothèse : et M.H. renvoie avec raison aux courses de filles à Sparte et à Olympie qui sont évidemment les exemples mieux connus, mais non les seuls. Dès sa propre autoanalyse dans sa contribution au Bulletin épigraphique de la REG (1995), M.H. ajoutait à ses références de la p. 32 ce complément qu'il devait à Fr. Graf lui signalant que Chios connaissait des concours gymniques qui voyaient la participation de garçons et de filles ${ }^{17}$. Un commentaire culturel de ces realia cultuels chiotes se trouve dans un passage d'Athénée qui confirme qu'à Chios, aux « jeux gymniques et aux courses à pied,... on voit rivaliser entre eux les jeunes gens des deux sexes » et que c'est « un vrai plaisir » (XIII, 2018,566 d). Le passage est en effet intégré à l'éloge que fait l'un des sophistes de la beauté. Il y est entre autres question de concours pour sélectionner sur des critères esthétiques ceux qui seront chargés de porter, dans les processions, les objets du culte et l'on s'y félicite de la bonne « habitude qu'ont les Spartiates de montrer nues leurs jeunes filles aux étrangers $»^{19}$. Cette beauté des filles, en de telles circonstances, on sait bien ce qu'elle contient d'indication d'âge. Être 'belle', c'est être en âge, en âge de développement pubertaire suffisant pour être désirée par l'homme.

16 M.H. renvoie là à sa contribution au colloque de 1990 sur la Thessalie (Thessalie et Macédoine: affinités et convergences, in La Thessalie: 15 années de recherches [1975 - 1990], bilans et perspectives Lyon, 17 - 22 auril 1990, vol. 2, Athènes 1994, p. 249-254). Cette conclusion historique est élargie p. 122

17 Nordionische Kulte, Rome, 1985, p. 444, I. Cbios, $\mathrm{n}^{\circ} 41$.

18 Je corrige M.H. dans $B E$ (1995), p. 405.

19 Il y a tout un dossier maintenant bien cohérent de la nudité (ou de la semi-nudité) rituelle chez les filles dans le passage d'âge. On se réfèrera à N. SERWINT, The Female Atbletic Costume at the Heraia and Prenuptial Initiation Rites, in AJA, 97 [1993], p. 403-422. Sur le sein droit dénudé, voir P. BRUlé, Héraclès et Augé. À propos d'origines rituelles du mythe, in Héraclès et le féminin. Actes du Colloque de Grenoble, 22-23 octobre 1992, Bruxelles-Rome, 1996 (Institut bistorique belge de Rome, XXXI), p. 47. 
Rappelons, parmi d'autres justifications de cette interprétation, cet extrait d'une inscription athénienne qui décrit quel choix il convient d'opérer entre les filles pour les fonctions d'ergastines (celles qui participent au tissage du péplos d'Athéna) : elles seront ai kalai tôn partbenôn: celles qui, parmi les partbenoi, sont 'belles'. Rappelons aussi cette façon qu'a d'Aristophane de décrire cette autre fille se trouvant dans le pro tou gamou pubère, la canéphore : c'est « devenue belle ${ }^{20}$ qu'elle a porté-la-corbeille. La plastique, l'esthétique qui satisfont les dieux, varient en même temps que la biologie : est belle celle qui est en âge. ${ }^{21}$. Voilà comment on doit imaginer les néai d'Hésychius, voilà dans quel état sont aussi, je pense, les filles qui ont-fait-leurjeunesse, ou, pour être plus précis, celles pour qui, parmi elles, on offre ces dédicaces.

Mais une difficulté surgit : si le caractère virginal des filles pubères dans les services divins de nos dossiers des rites de la jeunesse est assuré, en toute rigueur, rien ne dit que tout à la fois les 'nouvelles' de la notice d'Hésychius et les Thessaliennes et les Macédoniennes qui nebeusasan fussent des partbénoi. Or ce que nous venons de dire de la 'beauté' s'applique aussi à la nymphè si l'on prend garde à la rapidité du passage entre l'enfance et l'état d'épouse en Grèce. Les filles des dossiers de M.H. pourraient tout aussi bien être de jeunes mariées.

\section{Une « rançon »}

Celle dont P. Clément parlait surtout - parce qu'elle était une des seules à être connue en 1934 et parce que sa dédicace est un peu plus loquace - c'était cette Eubiotéia pour laquelle Hippolochos verse une « rançon ». Qu'est-ce que cela signifie?

Il devait trouver un appui à son interprétation dans le fait que cette Eubiotéia était un peu connue. Grâce, en effet, à la publication en 1927-1928 par N.I. Giannopoulos ${ }^{22}$ d'un décret du peuple de Larissa, il apparaissait qu'Eubiotéia avait été ensuite prêtresse (de qui ? d'Artémis Throsia ?), puis qu'elle était devenue l'épouse de cet Hippolochos (membre d'une famille connue de Larissa - les Hippolochoi -, comme l'est celle d'Eubiotéia - les Alexippoi) qui avait payé les lutra, et offert la dédicace. Cela faisait beaucoup, et ce faisceau de renseignements avait amené P. Clément à interpréter le versement d'une "rançon " comme le rachat à la divinité d'une fille qui s'était trouvée alors dans sa dépendance, selon un procédé qui ressemble à un affranchissement. Arvanitopoulos, Papachatzis et Helly ont compris cette « rançon » comme une action de grâce ou une offrande de propitiation à destination d'Artémis Throsia dans les vertus qu'ils prêtent à cette déesse, celle d'une divinité des accouchements. Mais laissons pour l'instant de côté cette question des fonctions des divinités évoquées dans le dossier.

20 Pais kalè : Lysistrata, 646.

21 Sur la 'beauté' comme âge féminin, P. Brulê, La fille d'Athènes, Paris, 1987, p. 297, 301 sq., 306, 316; sur tout cela L. BRUIT ZAIDMAN est revenue (Les jeunes filles dans la cité grecque, in Clio, Histoire, femmes et sociétés, 4 [1996], p. 33-50, ici p. 39).

22 Deltion, 11 (1927-1928), p. 55-56. 
M.H. retrouve lutron ou lutra ${ }^{23}$ dans une inscription mentionnant deux dédicantes à Artémis Akraia à Tricca (p. 34) et dans une autre émanant d'un certain Kratérophon pour les Dioscures (p. 38).

Pour avancer dans l'interprétation, M.H. produit encore d'autres pièces à conviction avec un terme qu'il juge proche de lutron-lutra. Ainsi cette dédicace p. 30 qui appartient à un lot homogène du Musée de Larissa en provenance, pour les deux premières, d'Atrax, pour la troisième, d'un site non identifié,

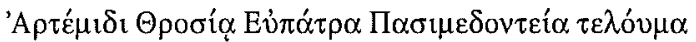

Et puis cette autre, p. 40 (de Phalanna):

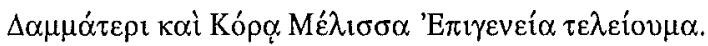

La place occupée par teleouma dans ces dédicaces, le fait que, dans l'une d'elles, la divinité soit la même que celle à laquelle on paie une « rançon » (Artémis Throsia) invitent à comparer / rapprocher ce terme de lutron. Le sens en est assez clair : «teleouma : dedication on the occasion of teleiôsis» dit le Liddell-Scott, qui ajoute pour " teleiôsis : development, completion of physical growth "; la dérivation à partir de teleios renvoie à l'idée d'accomplissement, de maturité. Le versant symbolique nous en est bien connu depuis longtemps à travers le couple parfait appartenant aux Theo $i$ gamèlioi tels que nous les présente Plutarque: Zeus Téléios et Héra Téléia ${ }^{24}$. Le teleouma offert par Eupatra fille de Pasimédon témoigne donc très probablement de la maturation biologique de son corps. Ce sens de don, d'offrande, au moment où se vérifie la maturité du corps s'accorde bien avec l'interprétation que M.H. propose du participe nebeusasa. Si cela, comme je le crois, est juste, il y a, dans lutron quelque chose d'assez irréductible à cette idée, c'est la notion de contrainte. Un sens proche peut-être, mais non pas identique. Or, le formulaire des dédicaces invite évidemment au rapprochement. $X$ [dédie ceci] à Artémis Throsia ou à Déméter et Koré à l'occasion de sa maturation physique.

Le sens de lutron et lutra tel qu'il est habituellement rendu me reste en partie imperméable dans ce contexte, comme me semble un peu outré le commentaire que fait M.H. de l'usage de ce termes dans les dédicaces. Voici ce qu'il écrit p. 39 à propos « de la nebeusasa Eubiotéia »: après son stage initiatique, au moment où elle est devenue une "femme ${ }^{25}$ accomplie », "son fiancé (ou déjà mari) Hippolochos dédie pour elle la rançon de sa liberte $e^{26} »$. Le fait que ces textes empruntent au vocabulaire des affranchissements ${ }^{27}$ explique sans doute la coloration très « hiérodule » de ce commentaire. Pourtant, et pour autant que je sache, ce serait la première fois que nous rencontrerions dans ces usages initiatiques - qu'ils soient féminins ou masculins -

23 Construit sans article comme dans tous les actes d'affranchissement thessaliens que vient de rassembler A. BIELMAN (Lutra, prisonniers et affranchis, in $M H, 46,1$ [1989], p. 25-41), à la différence des textes delphiques (respectivement p. 26-27 et p. 28).

24 À cette nuance près que je ne trouve pas exprimée chez M.H. p. 39 ou p. 40 que le teleouma, comme les lutra, s'appliquent à une fille non mariée. C'est l'homme qui fait la mariće. Ce Zeus et cette Héra la concerneront plus tard.

25 Femme, mais non épouse, si nous comprenons bien.

26

C'est moi qui souligne

27 Voir note 21 
l'idée d'une privation de liberté. Qu'il s'agisse de réclusion - ce qui peut être le cas des arrhéphores qui « vivent un certain temps auprès de la déesse » dans un parthenônou qu'il s'agisse de séclusion - comme on peut l'imaginer pour les ourses dans un sanctuaire important comme Brauron -, la terminologie servile n'est jamais employée à ma connaissance ${ }^{28}$, en tout cas dans nos dossiers extra-macédoniens. Certes, d'autres documents (surtout en provenance de Béroia) publiés dans le dossier 7 par M.H. témoignent de l'existence de consécrations de jeunes à des divinités (surtout des courotrophes). Mais il convient enfin de relever le caractère fort tardif des consécrations d'enfants libres à la divinité, phénomène auquel sont consacrés les dossiers 4, 5 et 7. Je crois qu'entre la consécration de jeunes (ou très jeunes) hiérodules à l'époque impériale (jusqu'au III $^{e}$ siècle ap. J.-C.) et les services religieux initiatiques des filles d'Athènes ou de Sparte aux époques archaïque et classique, il existe de telles différences de conception qu'il est difficile de prendre appui sur une institution pour interpréter l'autre. Si l'origine en est la même - ce qui n'est pas absolument assuré - le sens a beaucoup changé. Je ne me résous donc pas aisément à croire qu'Eubiotéia fut un temps privée de liberté. Ce qui me reste encore énigmatique, c'est le parallèle qu'établissent les documents eux-mêmes entre lutron et teleouma.

Quoi qu'il en soit, il me semble que cet usage de payer une « rançon » à la divinité regarde plus vers l'avenir que vers le passé. Je crois que cette idée de « rançon » à payer à Artémis Throsia quand en vient le temps a un fondement profondément religieux et qu'elle provient non de ce qu'il faudrait libérer la fille d'une éventuelle sujétion à la divinité (dont ces textes ne disent rien), mais de ce qui l'attend: une impureté à venir. Le fait que ce soit celui dont on sait qu'il est son futur mari qui ait versé la "rançon » me semble militer pour cette explication. Il faut renvoyer là à deux scholies au texte des Magiciennes de Théocrite ${ }^{29}$ et à un passage de Libanios $(5,1)$ que j'avais déjà utilisés dans l'étude des services pubères pro tou gamou. Ces passages mettent explicitement en rapport un service divin pubertaire : la canéphorie, avec le mariage et la perte de la virginité. Le fait qu'il s'agisse de la canéphorie, loin d'opposer cet exemple athénien à celui des filles ou des jeunes femmes qui nebeusasai, les en rapproche au contraire. D'abord en raison de la très étroite parenté entre la "beauté - développement physique " de la canéphore et de celle qui nebeusasa. Ensuite parce que la canéphorie dont parlent ces textes est une canéphorie pour Artémis (ce qui n'est pas si usité). Ils disent que «celles qui sont sur le point d'être prises pour épouses font les canéphores afin d'être purifiées de leur virginité », c'est-à-dire de la perte à venir de leur virginité. La protection d'Artémis, dit Libanios, vaut pour l'avenir, elle doit profiter « à celles qui

28 Les parallèles sur lesquels M.H. prend appui, p. 117, ne me semblent pas convaincants non plus. Ainsi, le fait, pour les ourses de Brauron, parallèlement au port de la crocote, à la mimésis de l'ourse, de devoir periepein to bieron (schol. Arist., Lys., 645) n'implique pas que leur séjour soit considéré comme marquant le moins du monde une perte de statut. Rappelons à leur propos qu'un psépbisma perdu de la collection rassemblée par Kratéros dans la seconde moitié du IV ${ }^{\mathrm{e}}$ siècle (làdessus Jacoby, FGrH, 11 b, 1955, p. 94 sq.), cité dans une notice d'Harpocration citant elle-même Lysias ( $s . v$. arkteusai), semble indiquer que toutes les filles d'Athènes faisaient l'ourse; mais le débat n'est pas clos sur cette question puisque d'autres sources comme la scholie L1 (Leyden) (Arist., Ly's. 645) évoquent des epilegomenai parthenoi ( $f$. A. BRELICH, Paides e parthenoi, Rome, 1969, 263 sq.); j'ai rassemblé dans la conclusion de La fille d'Atbènes, p. 391 sq., les éléments pour une discussion sur les modes de recrutement des desservantes qui, tout à la fois, s'inscrivent dans le cadre des institutions politiques (la tribu comme à Cos et à Athènes) et dans ce qu'on ne peut appeler qu'un « choix eugénique » compte tenu de l'instance des sources sur cette question de l'eugenéia.

29 Ed. Wendel, 284, 2. 
accèdent aux relations amoureuses $»^{30}$. En Thessalie comme ailleurs, celles qui atteignent un certain développement physique, qui deviennent 'belles' et qui sont sur le point d'être «prises par un homme », celles-là doivent dédier quelque chose à Artémis pour se propitier sa protection et pour se faire pardonner à l'avance la perte de leur virginité. On aurait donc affaire, si je ne me trompe pas, à un usage métaphorique de lutron.

\section{Le divin}

Nous savons qui elle est, ce qu'elle vit et ce qu'elle craint, voyons maintenant qui, dans la surnature, la conduit et la protège.

C'est Artémis Throsia qui est citée le plus souvent (textes des p. 25 et 26; 3 textes p. 30 (dont le dernier mentionne le teleouma]). L'interprétation de cette épiclèse a elle aussi posé des problèmes aux exégètes. Arvanitopoulos avait proposé de rattacher le terme à la racine tbor- 'enfler' et, à partir de l'idée du gonflement des bourgeons, de faire de cette Artémis « une déesse de la végétation, de la naissance, de la croissance des bébés »; P. Clément lui emboîte le pas et emploie l'expression de « déesse de la fertilité » pour cette Throsia qu'il juge analogue aux Artémis Généteira, Lochia ou Eileithyia que l'on trouve à Gonnoi.

Continuons à présenter les diverses épiclèses artémisiaques pour revenir ensuite à Throsia. Il y a aussi une épiclèse d'un genre assez commun chez Artémis, qui est l'épiclèse topographique: la Dynatis qui nebeusasa à Volo (IG) faisait sa dédicace à une Pagasitis. Rien à tirer de ce renseignement puisque les fonctions et l'être-au-monde de ces Artémis topographiques sont spécifiques et ne se laissent découvrir que lorsque le hasard nous a légué une documentation suffisante (Brauronia, Aulidéia, Mounichia, Amarynthia...).

Il reste que cette dédicace de deux jeunes filles mentionnant une « rançon » à Tricca est destinée à une Artémis Akraia. Le commentaire de M.H. sur le rapport éventuel de cette épiclèse à l'Héra Akraia bien connue à Corinthe me semble judicieux. La définition habituelle d'Akraia comme 'de la hauteur', 'de la cime' n'est pas sûre, là comme ailleurs. C'est akrios-akria qui sont utilisés dans ce sens là (par ex. à Argos (Hés. s.v.) pour Artémis, Athéna et Aphrodite). Et M.H. indique donc une autre piste, celle d'une glose de l'Etymologicum Magum et une autre d'Hésychius où akraia est défini comme une "pais thèleia chez les Macédoniens ». Nous voilà ramenés aux mêmes notions, à la même ambiance, celle de la jeunesse féminine. Avec A. Fick, M.H. glose l'akraia macédonienne comme « celle qui atteint la fleur de l'âge » (p. 37).

Voilà qui est significatif. Et c'est tant mieux parce que Throsia, de son côté, continue à me poser des problèmes. En effet, si la formation de l'épiclèse remonte bien, comme il semble (Chantraine; p. 33), à la racine thrô-, son sens nous entraîne aussi bien vers 'sauter', 'bondir', 's'avancer vivement', que vers 'féconder', 'saillir'. Il me semble assez clair que le lien entre les deux séries se trouve dans l'observation du monde animal, et en particulier, de la reproduction de certaines espèces domestiques, spécialement des ovins au cours de ce qu'on appelle la lutte en français, et qui s'avère être proprement une saillie bondissante. Le terme se retrouve en des textes variés à 
connotation fréquemment religieuse avec les deux sens : celui du 'bond' dans le contexte bachique bien connu du bain dans le lait dans les lamelles de Pélinna (ethores aux 1.3 et 4 ) et dans celui de 'saillie' dans l'Hymne à Zeus de Palaiokastro (etbores, p. 160-162 Powell) ${ }^{31}$ et dans le poème orphique cité dans le $P$. Derv. col. XIV, 4 où il s'agit du jaillissement (sans doute du pénis) dans l'éther. Il y a dans cette acception sexuelle du verbe comme un non-sens artémisiaque qui la rend très difficilement imaginable. Je sais bien qu'il y a dans le polythéisme une invention de détail qui rend possibles des Aphrodite vierges, des Arès efféminés, des Athéna mères, mais l'évidence est trop grande à côté pour être délaissée. Throsia doit plus probablement être entendue dans le premier sens évoqué plus haut 'sauter', 'bondir', 'avancer vivement', sens convenable pour le principal exercice de celles qui sont alors dédiées à ladite Artémis : la course. Si cette interprétation est juste, cette épiclèse trouverait son origine dans le gestuel de la course lui-même. Une épiclèse qui prendrait donc tout son sens dans le gestuel du rite.

Malgré ces quelques difficultés, tout va bien avec Artémis; nous sommes, par rapport au reste de l'Hellade, en divinité de connaissance. Tout (ou presque) de ce qu'on sait des initiations de la jeunesse féminine, que ce soit attique ou péloponnésien (95\% du dossier), tout nous renvoie à elle et à Athéna. Aussi la grande difficulté de ce livre se trouve-t-elle dans l'étrange présence de Déméter.

1- Si certaines dédient (ou font dédier) à Artémis Throsia des témoignages de leur nouvelle maturité (teleouma), d'autres comme cette Mélissa de Phalanna (?) (IG, IX 2 , 1235, p. 40; ci-dessus, p. 326) le font à Déméter et Koré. 2-C'est dans le sanctuaire de Déméter et Koré près de Létè («sur les collines qui surplombent à l'ouest le défilé de Dervini ») qu'ont été découvertes 1- une dédicace datée de la prêtrise de Bérénika par trois jeunes filles ou jeunes femmes archineusasai (p. 44; ci-dessus p. 323) consacrée à Déméter; 2- une autre, dédiée par une Alexandra qui simplement neusasa, est faite à Kala Théa 'La Belle Déesse' (p. 44). Quant aux autres textes en provenance du sanctuaire et que M.H. publie dans le dossier 3 (p. 55-58), ils ne nomment pas Déméter et ne font référence qu'à des courses de garçons. Et dire que des courses de filles avaient lieu dans ce même sanctuaire de Déméter et Koré à Létè, c'est s'appuyer in fine sur la seule notice d'Hésychius. On dira, possible, probable même, mais non assuré.

Quoi qu'il en soit, l'essentiel n'est pas là. Comment justifier cette énorme exception culturelle qui ferait de Déméter, partout et toujours associée à la sexualité féminine adulte, la protectrice-conductrice du passage pubertaire?

Deux solutions, deux explications peuvent être proposées. Permanence du signifiant, variation du signifié. Malgré la rareté (soulignée par M.H., p. 114) du culte de Déméter en Macédoine, nous pourrions avoir affaire localement à une de ces divinités attrape-tout comme on en rencontre ici et là. Une divinité topique - on dira comme Héra à Samos ou Argos, comme Athéna à Athènes - comme celles qui débordent

31 Rapprochement de G. RiCCIARDELLI APICELLA, Le lamelle di Pelinna, in SMSR, 58 (1992), p. 27-39 cité par Cl. CALAME, Invocations et commentaires orpbiques : transpositions funéraires de discours religieux, in Discours religieux dans l'Antiquité, Actes du colloque Besançon 27-28 janvier 1995, Paris, 1995, p. 18 sq.; sur 'bondir', 'saillir', cf. l'article de P. LÉVÊQUE supra, p. 239. 
largement de leurs fonctions habituelles panhelléniques pour capter à leur profit des fonctions normalement dévolues ailleurs à d'autres secteurs du polythéisme ${ }^{32}$.

La seconde interprétation suggérée par M.H. (p. 50) consisterait à identifier la Belle Déesse des inscriptions avec Koré. Dans cette perspective, les épreuves de passage pubère ou de sortie de puberté pourraient lui être normalement consacrées. Koré, comme pôle virginal, mais aussi comme modèle de la pais séductrice, prise par son époux quand vient l'âge grec du mariage. C'est le regard tourné vers le passé récent. Pôle matrimonial de Déméter qui intéresse évidemment les hommes; d'où le rôle d'un Hippolochos. C'est le regard tourné vers le thalamos... Il faudrait alors revenir sur cette remarque de la p. 325 qui note que le dossier n'interdit pas que l'on pense à la nymphè.

Tout en précisant que les deux interprétations ne s'excluent pas, on conclura en disant qu'il nous est bien difficile de démêler le probable du possible; les sources en sont la cause qui, quoique précieuses et nouvelles, nous interdisent, de façon irritante, les recoupements indispensables.

32 Pages décisives sur ce processus de Cl. CALAME, Les cbours de jeunes filles en Grèce arcbaïque, I, 1977, p. 222, repris dans P. Brulé, La fille d'Athènes, p. 221. 